\title{
Documento de consenso sobre el uso de los iSGLT2 en el tratamiento de pacientes con diabetes mellitus tipo 2
}

\author{
Juan C. Garnica-Cuéllar', Fernando J. Lavalle-González², José A. Magaña-Serrano,

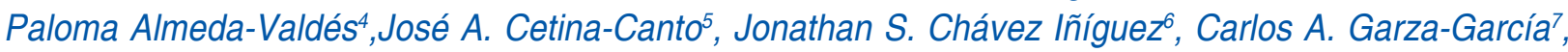 \\ Antonio González-Chávez ${ }^{8}$, Guillermo González-Gálvez G9 ${ }^{9}$, Juan H. Medina-Chávez ${ }^{10}$, \\ Gela Pimentel-Morales ${ }^{11}$ y Valentín Sánchez-Pedraza ${ }^{12}$
}

'Departamento de Endocrinología del Centro Médico Nacional 20 de noviembre, Instituto de Seguridad y Servicios Sociales de los Trabajadores del Estado, Ciudad de México, Méx.; ${ }^{2}$ Clínica de Diabetes HU, Hospital Universitario Dr. José E. González, Universidad Autónoma de Nuevo León, Monterrey, N.L.; ${ }^{3}$ División de Insuficiencia Cardiaca y Trasplante, Hospital de Cardiología, Centro Médico Nacional Siglo XXI, Instituto Mexicano del Seguro Social, Ciudad de México, Méx.; ${ }^{4}$ Departamento de Endocrinología y Metabolismo, Unidad de Investigación de Enfermedades Metabólicas, Instituto Nacional de Ciencias Médicas y Nutrición Salvador Zubirán, Ciudad de México, Méx.; ${ }^{5}$ Departamento de Medicina Interna y Endocrinología, Hospital Regional, Instituto de Seguridad y Servicios Sociales de los Trabajadores del Estado, Mérida, Yuc.; ${ }^{6}$ Departamento de Nefrología, Hospital Civil de Guadalajara Fray Antonio Alcalde, Guadalajara, Jal.; 'Departamento de Nefrología, Clínica RENALL MTY, Monterrey, N.L.; ${ }^{8}$ Clínica para la Atención Integral del Paciente con Diabetes y Obesidad, Hospital General de México Dr Eduardo Liceaga, Ciudad de México, Méx.; ${ }^{9}$ Servicio de Endocrinología, Hospital Civil de Guadalajara Dr. Juan I. Menchaca, Guadalajara, Jal.; ${ }^{10}$ Departamento de Medicina Interna y Geriatría, Coordinación Técnica de Excelencia, Clínica Instituto Mexicano del Seguro Social, Ciudad de México, Méx.; ${ }^{11}$ Servicio de Hemodinámica, Cardiología Clínica y Cardiología, Hospital de Cardiología Centro Médico Nacional Siclo XXI, Instituto Mexicano del Seguro Social, Ciudad de México, Méx.; ${ }^{12}$ Servicio de Endocrinología, Hospital General de México Dr. Eduardo Liceaga, Ciudad de México, Méx. México

\section{Resumen}

Aunque en los últimos años en México ha mejorado la calidad de la atención de la diabetes mellitus (DM) y ha aumentado el acceso a servicios de salud y medicamentos, existe una falta de apego a las recomendaciones de las guías de práctica clínica, que podría explicar la falta de un control glucémico adecuado en muchos de los pacientes con DM. Los inhibidores del cotransportador de sodio-glucosa tipo 2 (iSGLT2) han sido la última clase de agentes antidiabéticos en recibir la aprobación de la Food and Drug Administration (FDA) y de la Comisión Federal para la Protección contra Riesgos Sanitarios de México (COFEPRIS). Con el fin de mejorar el uso de los iSGLT2 en la práctica clínica en México, en este documento se presentan las recomendaciones emitidas por un panel de 11 expertos mexicanos con base en las nuevas evidencias publicadas para el tratamiento de los pacientes con DM2.

PALABRAS CLAVE: Diabetes mellitus tipo 2. iSGLT2. México.

\section{Consensus on the use of iSGLT2 in the treatment of patients with type 2 diabetes mellitus}

\begin{abstract}
Although in recent years in Mexico the quality of diabetes mellitus (DM) care has improved and access to health services and medications has increased, there is a lack of adherence to the recommendations of the clinical guidelines, which could explain the poor glycemic control in many of the patients with DM. Sodium-glucose cotransporter type 2 (iSGLT2) inhibitors have been the last class of antidiabetic agents to receive approval from the Food and Drug Administration (FDA) and COFEPRIS (Mexico). In order to improve the use of SGLT2i in clinical practice in Mexico, this paper presents the recommendations issued by a panel of eleven Mexican experts based on the new published evidence for the treatment of patients with DM2.
\end{abstract}

KEYWORDS: Type 2 diabetes mellitus. SGLT2i. Mexico.

Correspondencia:

Fernando Lavalle-González

E-mail: fernando.lavallegn@uanl.edu.mx

0016-3813/๑ 2021 Academia Nacional de Medicina de México, A.C. Publicado por Permanyer. Este es un artículo open access bajo la licencia CC BY-NC-ND (http://creativecommons.org/licenses/by-nc-nd/4.0/).
Fecha de recepción: 09-11-2021

Fecha de aceptación: 10-11-2021

A.C. Publicado por Permanyer. Este es un artículo open access bajo la licencia 


\section{Introducción}

La diabetes mellitus tipo 2 (DM2) es un trastorno metabólico crónico caracterizado por hiperglucemia persistente, que ha alcanzado en todo el mundo proporciones epidémicas y se ha convertido en un problema de salud pública de primera magnitud. Aproximadamente 415 millones de adultos entre las edades de 20 a 79 años tenían DM en 2015, y se espera que este número aumente a otros 200 millones para $2040^{1,2}$.

En México, datos de las Encuestas Nacionales de Salud y Nutrición (ENSANUT) revelan que la DM ha seguido una tendencia ascendente y ha experimentado un rápido aumento de la prevalencia en los últimos años, pasando del $4.6 \%$ de personas mayores de 20 años con diagnóstico conocido en el año 2000 hasta el $10.4 \%$ en 2018. Esto representa un total de 8.6 millones de personas con diagnóstico de DM. Las proyecciones a 25 años indican que en 2045 en torno al $20 \%$ de la población mexicana podría desarrollar $\mathrm{DM}^{3}$.

La hiperglucemia crónica en sinergia con otras alteraciones metabólicas en pacientes con DM puede causar daño a varios sistemas de órganos, lo que lleva al desarrollo de complicaciones de salud discapacitantes y potencialmente mortales, las más prominentes de las cuales son microvasculares (retinopatía, nefropatía y neuropatía) y complicaciones macrovasculares que conducen a un riesgo de enfermedades cardiovasculares (ECV) de dos a cuatro veces mayor ${ }^{1}$.

En países desarrollados, el porcentaje de pacientes con control glucémico adecuado es del $50-60 \%$, a pesar de que la gran mayoría de pacientes recibe algún tratamiento para la DM. En México, las tasas de control glucémico adecuado han mejorado en los últimos años, pero aún siguen siendo muy bajas ${ }^{4}$.

En los últimos años también ha mejorado la calidad de la atención y ha aumentado el acceso a servicios de salud y medicamentos. Sin embargo, existe una falta de apego a las recomendaciones de las guías de práctica clínica, lo que dificulta el seguimiento del paciente y su adherencia al tratamiento, y podría explicar por qué no se ha logrado un control glucémico adecuado en muchos de los pacientes con $\mathrm{DM}^{4}$.

Los agentes terapéuticos que controlan la hiperglucemia se pueden incluir en uno de cinco grupos: a) sensibilizadores de insulina (metformina y pioglitazona); b) secretagogos de insulina (insulina, sulfonilureas y meglitinidas); c) terapias basadas en incretinas (agonistas del receptor del péptido 1 similar al glucagón [AR-GLP ${ }^{1}$ e inhibidores de la dipeptidil peptidasa-4 [iDPP4); d) inhibidores de la absorción de glucosa gastrointestinal (acarbosa), y e) inhibidores de la reabsorción renal de glucosa (inhibidores del cotransportador de sodio-glucosa tipo 2 [iSGLT ${ }^{2}$ ). Los iSGLT2 han sido la última clase de agentes antidiabéticos en recibir la aprobación de la Food and Drug Administration (FDA) y de la Comisión Federal para la Protección contra Riesgos Sanitarios de México (COFEPRIS) $)^{5,6}$.

Los iSGLT2 son una clase de antidiabéticos que bloquean la reabsorción tubular renal de glucosa con distintos beneficios metabólicos y hemodinámicos multisistémicos. En los últimos años, en México se han aprobado tres iSGLT2: canagliflozina, dapagliflozina y empagliflozina.

Además de la excreción urinaria de glucosa, que tiene una consecuencia directa sobre la disminución de la hemoglobina glucosilada (HbA1c), el peso y la glucemia en ayunas, los iSGLT2, debido a la diuresis osmótica, también pueden proporcionar una mayor reducción de la presión arterial, sin que por ello se vea afectada la frecuencia cardiaca de los pacientes ${ }^{7}$.

En este documento se presentan las recomendaciones emitidas por un panel de 11 expertos mexicanos con base en las nuevas evidencias publicadas sobre el uso de los iSGLT2 en pacientes con DM2.

\section{Control de la glucosa}

El parámetro más utilizado para valorar el control glucémico en la DM2 es la HbA1c. Un metaanálisis de los estudios Action to Control Cardiovascular Risk in Diabetes (ACCORD), Action in Diabetes and Vascular Disease: Preterax and Diamicron Modified Release Controlled Evaluation (ADVANCE) y Veterans Affairs Diabetes Trial (VADT) sugirió que en la DM2 una reducción de la $\mathrm{HbA} 1 \mathrm{c}$ del $1 \%$ se asocia con una reducción del riesgo relativo del $15 \%$ en infarto de miocardio (IM) no mortal, sin efectos benéficos en el accidente cerebrovascular, la ECV o la mortalidad por todas las causas u hospitalización por insuficiencia cardiaca (IC). El control intensivo de la glucosa fue beneficioso para los eventos cardiovasculares (CV) en pacientes con DM de corta duración, HbA1c más baja al inicio del estudio y sin ECV ${ }^{5,8-10}$.

Estas evidencias, junto con los resultados del análisis postintervención del estudio UKPDS (UK Prospective Diabetes Study), que demostró que, en 
pacientes con DM2 de reciente diagnóstico y sin complicaciones asociadas, el control glucémico intensivo reducía los eventos CV y las complicaciones microvasculares, subrayan la importancia del control glucémico adecuado desde las fases más tempranas de la DM2 para disminuir las complicaciones microvasculares y la morbimortalidad de origen $\mathrm{CV}^{5,8-10}$.

Los objetivos de HbA1c deben individualizarse. En general, se considera que debería alcanzarse una $\mathrm{HbA} 1 \mathrm{c}<7 \%(<53 \mathrm{mmol} / \mathrm{mol})$ en la mayoría de los pacientes. Sin embargo, los objetivos de $\mathrm{HbA} 1 \mathrm{c}$ podrían ser más estrictos $(<6.5 \%)$ en pacientes más jóvenes con una DM de corta duración y sin evidencia de ECV, si pueden lograrse sin hipoglucemia significativa. Objetivos de $\mathrm{HbA} 1 \mathrm{c}$ menos estrictos pueden ser adecuados para pacientes con antecedentes de hipoglucemia grave, esperanza de vida limitada, complicaciones microvasculares o macrovasculares avanzadas, fragilidad con múltiples comorbilidades o DM de larga duración $\mathrm{n}^{5,11}$.

Se ha informado que la variabilidad de la glucosa plasmática en ayunas es un fuerte predictor de la mortalidad por todas las causas y está relacionada con la ECV en pacientes con DM, lo que sugiere que el manejo de la variabilidad de la glucosa puede convertirse en un objetivo adicional ${ }^{5}$.

Los fármacos que reducen las excursiones de glucosa posprandial, incluidos los AR-GLP1, los iDPP4 y el iSGLT2 como canagliflozina (a dosis de $300 \mathrm{mg}$ ) parecen una forma atractiva de reducir la variabilidad de la glucosa ${ }^{5,12,13}$.

Las recomendaciones de la American Diabetes Association (ADA) 2021 consideran que el coeficiente de variación sea $<36 \%$, el tiempo en rango de la glucosa sea $>70 \%$ con una glucosa entre $70-180 \mathrm{mg} / \mathrm{dl}$ (tiempo < 5\% con glucosa $>250 \mathrm{mg} / \mathrm{dl}$, tiempo $<25 \%$ con glucosa en rango de $181-249 \mathrm{mg} / \mathrm{dl})^{14}$.

El tratamiento inicial recomendado por las guías de práctica clínica es monoterapia con metformina, si la $\mathrm{HbA1c}$ no es mayor $1.5 \%$ del valor meta propuesto, a menos que existan contraindicaciones, junto con modificaciones en el estilo de vida. Cuando esta estrategia no es suficiente para alcanzar los objetivos de HbA1c, o deja de serlo, deberá introducirse tratamiento combinado. La estrategia terapéutica depende del grado de glucotoxicidad, de modo que cuando la $\mathrm{HbA1c}$ es $\geq 10 \%$ se sugiere terapia con insulina más terapia oral o AR-GLP1. Las recomendaciones actuales han sido utilizar la adición gradual de medicamentos a la metformina para mantener la HbA1c en el objetivo, aunque algunos datos y recomendaciones de guías clínicas internacionales respaldan la terapia de combinación inicial cuando la HbA1c es 7.5-8\%, para lograr más rápidamente los objetivos glucémicos, particularmente para fenotipos específicos de pacientes según riesgo CV y enfermedad renal crónica (ERC), entre otros. Concretamente, las nuevas guías de la ADA de 2021 señalan que debe iniciarse terapia combinada en sujetos con $\mathrm{HbA} 1 \mathrm{c}$ 1.5\% arriba de la meta deseada ${ }^{5,14}$.

La combinación de metformina y un iSGLT2 ha demostrado no ser inferior a la combinación de metformina con sulfonilurea, y ser superior en terapia de adición vs. iDPP4 $4^{15,16}$.

\section{Recomendaciones}

- En pacientes en los que está indicado el control intensivo de la glucosa, se puede considerar como tratamiento de inicio metformina y un isGLT2, en aquellos de alto riesgo CV o ECV o enfermedad renal, ya que sus efectos son complementarios y van más allá del control de la glucosa, específicamente por sus beneficios en riesgo CV, reducción de la presión arterial, protección cardiorrenal y pérdida de peso.

- Existe acuerdo en que los iSGLT2 son una buena opción para reducir la variabilidad de la glucosa, ya que su mecanismo de acción independiente de la reducción de la glucosa vía insulina reduce las excursiones a lo largo del día, incluyendo la glucemia en ayuno y posprandial, con un perfil farmacológico de bajo riesgo para hipoglucemia.

- Los iSGLT2 pueden utilizarse en pacientes con resistencia a la insulina, ya que la disminución de la glucosa por un mecanismo no dependiente de insulina hace que mejore la capacidad de secreción de insulina al eliminar el fenómeno de glucotoxicidad e, indirectamente, mejora la resistencia a la insulina. Este mecanismo, junto con la reducción de peso, disminuye la resistencia a la insulina.

\section{Riesgo cardiovascular}

La DM2 se asocia con un aumento de la morbilidad y mortalidad CV. Los pacientes con DM2 tienen un aumento de dos a cuatro veces en el riesgo de ECV, que incluye enfermedad coronaria, accidente cerebrovascular isquémico y enfermedad arterial periférica, con un aumento de 1.5 a 3.6 veces en la mortalidad. La DM2 también es un factor de riesgo importante de IC, que afecta la calidad y la esperanza de vida de los pacientes ${ }^{14,17}$. 
Las condiciones que coexisten habitualmente con la DM2, como hipertensión y dislipidemia, son claros factores de riesgo para ECV, y la DM en sí misma confiere un riesgo independiente. Los iSGLT2 han demostrado beneficio en el contexto de DM2 e IC. Entre los mecanismos más aceptados para explicar el modo de acción del iSGLT2 en la IC se encuentran la mejora de la tensión parietal del ventrículo izquierdo secundaria a la disminución de la precarga (efecto de la natriuresis y diuresis osmótica) y la poscarga (mejora de la función endotelial y reducción de la sangre) $)^{18-20}$. Los mecanismos metabólicos incluyen la mejora del metabolismo y la bioenergética de los cardiomiocitos (mayor cetogénesis y mayor suministro de $\beta$-hidroxibutirato ${ }^{21}$, inhibición de la bomba de sodio-hidrógeno del miocardio (que conduce a una mayor concentración de calcio en las mitocondrias) ${ }^{22}$, reducción de la necrosis y fibrosis cardiacas (inhibición de la síntesis de colágeno ${ }^{23}$ y cambios en la producción de citocinas y tejido graso epicárdico ${ }^{24}$. Numerosos estudios han demostrado la eficacia de controlar los factores de riesgo CV individuales para prevenir o retrasar la ECV en personas con $\mathrm{DM}^{14}$.

Diversos ensayos controlados aleatorizados reportan reducciones estadísticamente significativas de los eventos CV en pacientes con DM2 y ECV establecida tratados con un iSGLT2. Los tres iSGLT2 aprobados en México, canagliflozina, dapagliflozina y empagliflozina, incluyen en sus fichas técnicas los beneficios CV después de la publicación de sus estudios de seguridad CV. La canagliflozina y la empagliflozina han demostrado beneficio en eventos adversos CV mayores (MACE) e IC. Con base en esta evidencia, la canagliflozina tiene indicación de prevención de MACE en población con DM2 y ECV establecida, la empagliflozina tiene indicación de prevención de la mortalidad en pacientes con DM2 con alto riesgo CV y la dapagliflozina tiene indicación para reducir el riesgo de hospitalización por IC en DM2 con ECV o con múltiples factores de riesgo $\mathrm{CV}^{7,14}$.

El Empagliflozin Cardiovascular Outcome Event Trial in Type 2 Diabetes Mellitus Patients (EMPA-REG OUTCOME $)^{25}$ fue un ensayo aleatorizado, doble ciego que evaluó el efecto de empagliflozina frente a placebo sobre desenlaces CV en 7,020 pacientes con DM2 y ECV. Los participantes del estudio tenían una edad media de 63 años, el 57\% tenía DM con más de 10 años desde el diagnóstico y el $99 \%$ tenía ECV establecida. EI EMPA-REG OUTCOME mostró que, durante una mediana de seguimiento de 3.1 años, el tratamiento redujo el desenlace combinado de IM, accidente cerebrovascular y muerte CV un $14 \%$, y la muerte CV un $38 \%$.

Con canagliflozina, dos grandes ensayos evaluaron por separado los efectos CV del tratamiento en pacientes con alto riesgo de eventos CV y el impacto de la terapia con canagliflozina en los resultados cardiorrenales en pacientes con ERC relacionada con la DM. El primero es el Canagliflozin Cardiovascular Assessment Study (CANVAS), que integró datos de dos estudios, el CANVAS y el CANVAS Renal $(\text { CANVAS-R })^{26}$. Combinando estos dos estudios, se incluyeron 10,142 participantes con DM2, que fueron asignados en forma aleatoria a canagliflozina o placebo y fueron seguidos durante un promedio de 3.6 años. La edad media de los pacientes fue de 63 años y el $66 \%$ tenía antecedentes de ECV. El análisis combinado de los dos ensayos encontró que la canagliflozina redujo en un $14 \%$ el desenlace combinado de muerte CV, IM o accidente cerebrovascular vs. placebo. Asimismo, encontró una reducción del 18\% en la subpoblación con ECV establecida ${ }^{27}$.

El segundo ensayo con canagliflozina es el Canagliflozin and Renal Events in Diabetes with Established Nephropathy Clinical Evaluation (CREDENCE) $)^{28}$, que asignó aleatoriamente a 4,401 pacientes con DM2 y ERC relacionada con la DM a canagliflozina $100 \mathrm{mg}$ al día o placebo. El desenlace primario fue una combinación de enfermedad renal terminal, duplicación de la creatinina sérica o muerte por causas renales o CV. El ensayo se detuvo anticipadamente debido a la evidencia concluyente de eficacia de canagliflozina, que logró una reducción del $30 \%$ en el riesgo de alcanzar el objetivo primario. También se observó una reducción del $20 \%$ en el compuesto de muerte CV, IM o accidente cerebrovascular, del $39 \%$ en hospitalización por IC y del 31\% en el compuesto de muerte CV u hospitalización por IC. Con dapagliflozina se llevó a cabo el ensayo Dapagliflozin Effect on Cardiovascular EventsThrombosis in Myocardial Infarction 58 (DECLARETIMI 58) ${ }^{29}$, que evaluó los efectos de dapagliflozina vs. placebo sobre los resultados CV y renales en 17,160 pacientes con DM2 y ECV establecida o múltiples factores de riesgo de ECV. Los participantes del estudio tenían una edad media de 64 años y el $40 \%$ de ellos tenían ECV establecida al inicio del estudio (el resto comprendía población de alto riesgo $\mathrm{CV}$ ), a diferencia de otros ensayos CV en los que la mayoría de los participantes tenían ECV establecida. El DECLARE-TIMI 58 cumplió con los criterios preespecificados de no inferioridad al placebo con respecto 
a MACE, pero no mostró una tasa más baja de MACE en comparación con placebo $(8.8 \%$ en el grupo de dapagliflozina y $9.4 \%$ en el grupo de placebo). Sin embargo, se observó una menor tasa del desenlace compuesto de muerte CV y hospitalización por IC del $17 \%$,11,14.

En resumen, existen evidencias de ensayos controlados aleatorizados que informan reducciones estadísticamente significativas en los eventos CV para canagliflozina y empagliflozina. Asimismo, existen evidencias sobre los beneficios de los iSGLT2 en la prevención de la hospitalización por IC $\mathrm{C}^{5,14}$.

Los beneficios CV de los iSGLT2 son independientes de la magnitud de la reducción de la glucosa y ocurren tempranamente como para ser atribuidos a la reducción de peso. En cuanto a la reducción de las hospitalizaciones por IC, parece ser el resultado de los efectos en parámetros hemodinámicos, como volumen plasmático reducido, efectos directos sobre el metabolismo y función cardiaca, natriuresis y otros efectos $\mathrm{CV}^{5}$.

Basándose en estos resultados, tanto la ADA como la European Society of Cardiology (ESC) recomiendan, en pacientes con ECV o alto riesgo CV, incluir un iSGLT2 o un AR-GLP1, o con IC incluir un iSGLT2 como parte del régimen de tratamiento antidiabético independientemente de la concentración de $\mathrm{HbA}_{1} \mathrm{c}^{5,14}$. Si el paciente ya está en tratamiento, pero no cuenta con un iSGLT2 o AR-GLP1, se recomienda considerar adicionar a uno de estos agentes con el fin de obtener el beneficio CV, o bien cambiar al iSGLT2 o el AR-GLP1 en los pacientes con ECV, aunque estén en cifras objetivo de $\mathrm{HbA1c}$, para obtener un beneficio $\mathrm{CV}$.

Recomendaciones

- En pacientes con DM y riesgo CV alto (si se utiliza la puntuación en enfermedad cardiovascular aterosclerótica [ASCVD] o Globorisk) o muy alto (si se utiliza la clasificación de la ESC) o con ECV establecida no tratados previamente, se recomienda considerar un iSGLT2 como una de la primeras opciones de tratamiento por los beneficios cardiorrenales demostrados.

- Se recomienda adicionar un iSGLT2 lo antes posible en pacientes con DM y riesgo CV alto (si se utiliza ASCVD o Globorisk) o muy alto (si se utiliza la clasificación de la ESC) o ECV establecida en tratamiento con cualquier otra clase terapéutica, independientemente de si alcanza 0 no el objetivo de $\mathrm{HbA1c}$, por los beneficios extraglucémicos que ofrecen los iSGLT2 (reducción de peso, disminución de la presión arterial, disminución de la progresión a ERC terminal).

- En pacientes con ECV se recomienda sustituir por otra terapia, si el paciente está con glitazonas o saxagliptina, por el riesgo de IC.

- En pacientes con DM y ECV establecida, se recomienda utilizar los iSGLT2 en estos escenarios mencionados (canagliflozina, dapagliflozina 0 empagliflozina), siguiendo la indicación para prescribir aprobada en México.

\section{Insuficiencia cardiaca}

Más allá de su potencial hipoglucemiante, se ha observado una reducción de la incidencia de IC y de hospitalizaciones por IC en pacientes con DM tratados con iSGLT2 ${ }^{30}$.

Se observaron reducciones en las hospitalizaciones por IC en EMPA-REG OUTCOME ${ }^{25}$, CANVAS $^{26}$ y DECLARE-TIMI $58^{29}$, incluidos los pacientes con y sin ECV aterosclerótica prevalente y aquellos con y sin antecedentes de IC. Resultados de estos ensayos con desenlaces CV y el CREDENCE ${ }^{28}$ revelaron beneficios más sólidos y consistentes en IC que en eventos CV mayores con iSGLT2. En el EMPA-REG OUTCOME, la adición de empagliflozina al tratamiento estándar condujo a una reducción significativa del 35\% en la hospitalización por IC en comparación con placebo. De manera similar, en el CANVAS y en el DECLARE-TIMI 58 hubo reducciones del 33 y $27 \%$ en la hospitalización por IC, respectivamente, con el uso de iSGLT2 frente a placebo. Los datos adicionales del ensayo CREDENCE con canagliflozina mostraron una reducción del $39 \%$ en la hospitalización por IC y una reducción del 31\% en la combinación de muerte CV u hospitalización por IC, en una población con nefropatía con albuminuria (relación albúmina: creatinina en orina [RACU] de 300 a $5,000 \mathrm{mg} / \mathrm{g}$ ).

El Dapagliflozin And Prevention of Adverse Results in Heart Failure (DAPA-HF) fue el primer ensayo clínico de un iSGLT2 para evaluar los eventos de IC como resultado primario e incluir participantes con y sin DM. En este ensayo se asignaron al azar 4,744 pacientes con IC clase II, III o IV de la New York Heart Association (NYHA) y una fracción de eyección del $40 \%$ o menos para recibir dapagliflozina (en una dosis de $10 \mathrm{mg}$ una vez al día) o placebo, además de la terapia recomendada. En el momento de la selección, el $42 \%$ de los pacientes de cada grupo tenían antecedentes de DM2 y un 3\% adicional de los pacientes de cada grupo fueron diagnosticados con DM. El 
objetivo primario fue una combinación de empeoramiento de la IC (hospitalización o una visita urgente que resultó en terapia intravenosa para la IC) o muerte CV. Con un seguimiento medio de 18.2 meses, la probabilidad de alcanzar el desenlace compuesto primario se redujo significativamente, un $16.3 \%$ para dapagliflozina en comparación con el $21.2 \%$ para placebo (razón de riesgo [HR]: 0.74; intervalo de confianza del 95\% [IC 95\%]: 0.65 a 0.85). Las tasas de eventos para los tres componentes del resultado compuesto fueron favorables a dapagliflozina, con una reducción del $30 \%$ en hospitalización por IC y del 18\% en muerte CV. Estos resultados fueron consistentes en todos los subgrupos, incluidos aquellos con y $\sin$ DM o ERC ${ }^{31}$.

Posteriormente, el Empagliflozin Outcome Trial in Patients with Chronic Heart Failure and a Reduced Ejection Fraction (EMPEROR-Reduced) ${ }^{32}$ evaluó también el desenlace primario de $\mathrm{IC} /$ muerte $\mathrm{CV}$, entre adultos con fracción de eyección reducida con y sin DM2. En el EMPEROR-Reduced, un total de 3,730 pacientes con IC de clase NYHA II, III o IV y una fracción de eyección del 40\% o menos fueron asignados aleatoriamente a recibir empagliflozina $(10 \mathrm{mg}$ una vez al día) o placebo, además de la terapia recomendada. El resultado primario fue la combinación de muerte CV y hospitalización por empeoramiento de la IC. Durante una mediana de seguimiento de 16 meses, la reducción en el resultado primario compuesto fue del $25 \%$, siendo el efecto de empagliflozina independiente de la presencia o no de DM. Sin embargo, un punto importante al analizar la muerte CV como componente del objetivo primario es que la empagliflozina no demostró disminuir la muerte CV (IC: 0.75-1.12) en esta población ${ }^{32}$.

Un metaanálisis de ambos ensayos mostró que el efecto del tratamiento estimado fue una reducción del $13 \%$ en la muerte por todas las causas y una reducción del 14\% en la muerte CV. La inhibición de SGLT2 estuvo acompañada de una reducción relativa del $26 \%$ en el riesgo combinado de muerte CV o primera hospitalización por IC, y por una disminución del $25 \%$ en la combinación de hospitalizaciones recurrentes por IC o muerte $\mathrm{CV}^{33}$.

Finalmente, el Evaluation of Ertugliflozin Efficacy and Safety Cardiovascular Outcomes Trial (VERTIS CV) demostró, en los pacientes tratados con ertugliflozina, una reducción del $30 \%$ en el riesgo de primera hospitalización por $\mathrm{IC}^{34}$.

Así pues, los iSGLT2 reducen las tasas de hospitalización por IC en pacientes de riesgo con DM2, independientemente de los antecedentes de ECV aterosclerótica o IC. Los pacientes seleccionados adecuadamente con DM2 y con alto riesgo CV pueden beneficiarse del inicio de los iSGLT2 para la prevención de la IC. Dado que solo una minoría de los pacientes (10 a 15\%) tenía un diagnóstico inicial de IC y la captura de datos de elementos clave relacionados con la IC (p. ej., fracción de eyección) fue incompleta en los desenlaces CV de los ensayos de iSGLT2 hasta la fecha, se están llevando a cabo varios ensayos clínicos específicos (NCT03036124; NCT03619213; NCT03057977; NCT03057951, y NCT03521934) para evaluar la utilidad de los iSGLT2 en el tratamiento de la IC manifiesta ${ }^{30}$.

Según la $A D A$ y la ESC, el tratamiento de primera línea en pacientes con DM2 e IC debe incluir un iSGLT2, incluso antes de metformina según la ESC ${ }^{5,14}$.

Recomendaciones

- En pacientes con DM e IC, pueden considerarse los tres iSGLT2 disponibles en México (dapagliflozina, empagliflozina y canagliflozina).

- Se recomienda el empleo de iSGLT2 con IC, ya que todos han demostrado reducir la hospitalización por IC y mortalidad CV.

\section{Enfermedad renal crónica}

Más del $40 \%$ de las personas con DM desarrollarán ERC, incluido un número significativo que desarrollará insuficiencia renal que requerirá diálisis y/o trasplante ${ }^{35}$.

Las guías KDIGO (Kidney Disease: Improving Global Outcomes) indican que el manejo de la glucemia para pacientes con DM2 y ERC debe incluir cambios en el estilo de vida, tratamiento de primera línea con metformina e iSGLT2, con terapia farmacológica adicional según sea necesario para el control de la glucemia ${ }^{35}$.

Se ha demostrado que los iSGLT2 previenen la progresión de enfermedad renal, especialmente en pacientes con tasa de filtrado glomerular estimada (TFGe) inicial conservada. Aunque los ensayos clínicos han incluido cohortes con TFGe tan baja como $30 \mathrm{ml} / \mathrm{min} / 1.73 \mathrm{~m}^{2}$, en México las aprobaciones para iniciar terapia con canagliflozina, dapagliflozina y empagliflozina es con TFGe > $30 \mathrm{ml} / \mathrm{min} / 1.73 \mathrm{~m}^{2}$ (fecha de aprobación: 22 de junio de 2021). Los iSGLT2 se están estudiando activamente en la ERC establecida (albuminuria) y los ensayos específicos en IC están inscribiendo pacientes con TFGe hasta $20 \mathrm{ml} / \mathrm{min} / 1.73 \mathrm{~m}^{2}$. Un ensayo de fase 3 (NCT02065791) 
que evaluó la canagliflozina en la DM2 y la ERC se detuvo recientemente de forma temprana por motivos de eficacia ${ }^{30}$.

La mayoría de los pacientes con DM2, ERC y una TFGe $\geq 30 \mathrm{ml} / \mathrm{min}$ por $1.73 \mathrm{~m}^{2}$ se beneficiarían del tratamiento con metformina y un iSGLT2. La metformina y los iSGLT2 reducen el riesgo de desarrollar complicaciones de la DM con un riesgo bajo de hipoglucemia. La eficacia en la reducción de la HbA1c con los iSGLT2 disminuye cuando la TFGe se encuentra entre $30-59 \mathrm{ml} / \mathrm{min} / 1.73 \mathrm{~m}^{2}$, pero tienen grandes efectos sobre la reducción de la progresión de la ERC y la ECV que parecen ser independientes de la TFGe. Se espera una disminución moderada de la TFGe (3 a $4 \mathrm{ml} / \mathrm{min} / 1.73 \mathrm{~m}^{2}$ ) después del inicio, pero las evidencias muestran que los iSGLT2 pueden tener un efecto nefroprotector a largo plazo, ya que en todos los estudios se demostró, además de beneficios en los desenlaces renales, una disminución en la pérdida progresiva de la TFGe menor y una reducción de la albuminuria 0 una disminución de la progresión o incremento de esta ${ }^{35}$.

En el EMPA-REG OUTCOME, el tratamiento con empagliflozina logró una reducción del $39 \%$ en el riesgo de aparición o empeoramiento de la nefropatía, del $38 \%$ en la progresión de la macroalbuminuria y del $44 \%$ en la duplicación de la creatinina sérica en pacientes con DM2 y enfermedad CV establecida. No hubo diferencias en la tasa de albuminuria incidente ${ }^{36}$.

En el CANVAS-R, los resultados mostraron un posible beneficio de canagliflozina con respecto a la progresión de la albuminuria, con una reducción del $27 \%$ (IC 95\%: 0.67 a 0.79) y el resultado combinado de una reducción sostenida del $40 \%$ en la TFGe, la necesidad de terapia de reemplazo, o muerte por causas renales (IC 95\%: 0.47 a 0.77$)^{26}$.

En el DECLARE-TIMI 58, el tratamiento con dapagliflozina se asoció con una reducción del $24 \%$ en el desenlace cardiorrenal secundario compuesto de disminución sostenida de al menos un $40 \%$ en la TFGe $\geq 60 \mathrm{ml} / \mathrm{min} / 1.73 \mathrm{~m}^{2}$, enfermedad renal en etapa terminal (definida como diálisis durante al menos 90 días, trasplante de riñón o TFGe $<15 \mathrm{ml} / \mathrm{min} / 1.73 \mathrm{~m}^{2}$ ), o muerte por causas renales o CV. En el desenlace compuesto excluyendo la muerte $\mathrm{CV}$, la reducción obtenida fue del $47 \%{ }^{29}$.

Además de las evidencias obtenidas a partir de los ensayos EMPA-REG con empagliflozina, CANVAS y CREDENCE con canagliflozina y DECLARE-TIMI 58 con dapagliflozina, se dispone de los resultados de un metaanálisis de EMPA-REG, CANVAS y DECLARETIMI-58 que estratificó subgrupos por ERC ${ }^{30}$; un metaanálisis de EMPA-REG OUTCOME, CANVAS, CREDENCE y DECLARE-TIMI 58 evaluando resultados renales ${ }^{37}$; y un metaanálisis de los ensayos DAPA-HF y EMPEROR-Reduced que evaluó el resultado primario de $\mathrm{CV} /$ muerte estratificados por TFGe $\left(<60 \mathrm{y} \geq 60 \mathrm{ml} / \mathrm{min} / 1.73 \mathrm{~m}^{2}\right)^{33}$.

En el primer metaanálisis, los iSGLT2 redujeron el riesgo de desenlaces renales adversos (empeoramiento de la insuficiencia renal, ERC terminal o muerte renal) en los pacientes con una TFGe de 30 $a<60 \mathrm{ml} / \mathrm{min} / 1.73 \mathrm{~m}^{2} 30$.

El segundo metaanálisis encontró que los iSGLT2 redujeron significativamente el riesgo de diálisis, trasplante de riñón o muerte renal en un 33\%, y hubo también reducción en ERC terminal y daño renal. Los beneficios de los iSGLT2 sobre los resultados renales se observaron en todos los subgrupos de TFGe, incluidos aquellos con TFGe entre $30-45 \mathrm{ml} / \mathrm{min} / 1.73 \mathrm{~m}^{237}$. En el tercero, la probabilidad de alcanzar el objetivo renal compuesto se redujo un $38 \%$ en los pacientes tratados con el iSGLT2 ${ }^{33}$.

El estudio CREDENCE, con canagliflozina, el cual se detuvo prematuramente por eficacia anticipada, fue el primer estudio publicado con un iSGLT2 en pacientes con ERC y DM2. El riesgo relativo del desenlace primario, el compuesto de enfermedad renal en etapa terminal (diálisis, trasplante 0 una TFGe sostenida $\left.<15 \mathrm{ml} / \mathrm{min} / 1.73 \mathrm{~m}^{2}\right)$, una duplicación del nivel de creatinina sérica o muerte por causas renales - CV fue un 30\% menor en el grupo de canagliflozina que en el grupo de placebo. En esta población se demostró que la canagliflozina disminuye los desenlaces renales y CV de manera segura ${ }^{28}$.

Recientemente, se han publicado los datos del DAPA-CKD, interrumpido prematuramente debido a la eficacia observada con dapagliflozina. En este estudio participaron 4,304 pacientes con una TFGe entre 25 y $75 \mathrm{ml} / \mathrm{min} / 1.73 \mathrm{~m}^{2}$ y una RACU de 200 a $5,000 \mathrm{mg} / \mathrm{g}$ para recibir dapagliflozina (10 $\mathrm{mg}$ una vez al día) o placebo. El resultado primario fue una combinación de una disminución sostenida en la TFGe de al menos un $50 \%$, ERC en etapa terminal o muerte por causas renales o $\mathrm{CV}^{38}$.

Con una mediana de seguimiento de 2.4 años, la probabilidad de alcanzar el desenlace primario se redujo un $44 \%$ en el grupo tratado con dapagliflozina. Los efectos fueron similares en pacientes con y sin $\mathrm{DM}^{38}$. 
Las últimas guías KDIGO señalan que en la mayoría de los pacientes con DM2, ERC y una TFGe $\geq 30 \mathrm{ml} / \mathrm{min} / 1.73 \mathrm{~m}^{2}$ se pueden utilizar juntos metformina y un iSGLT2 de forma segura y eficaz. De hecho, la mayoría de los participantes en los ensayos de resultados CV con iSGLT2 también fueron tratados con metformina, y muchos pacientes con DM2 requieren más de un antidiabético para alcanzar los objetivos glucémicos. La combinación de metformina y un iSGLT2 es conveniente porque tienen diferentes mecanismos de acción y ninguno conlleva un mayor riesgo de hipoglucemia. Incluso cuando se alcanzan los objetivos glucémicos con metformina, se debe agregar un iSGLT2 para lograr el beneficio en la reducción de la progresión de la ERC y la ECV ${ }^{35}$.

Los beneficios renales de los iSGLT2 parecen ser en gran medida independientes del control glucémico según una serie creciente de observaciones. Sus efectos hipoglucemiantes son modestos, especialmente en personas con TFGe de $45 \mathrm{ml} / \mathrm{min} / 1.73 \mathrm{~m}^{2}$. Sin embargo, la protección de los riñones es consistente y sustancial en esta población ${ }^{28}$. Los iSGLT2 también tienen beneficios renales en personas con glucemia bien controlada ( $\mathrm{HbA} 1 \mathrm{c}$ 7\%) en quienes la reducción adicional de la glucosa fue mínima ${ }^{39}$.

No hay evidencia con un diseño de ensayo clínico similar al estudio CREDENCE o DAPA-CKD con otros antidiabéticos que hayan demostrado protección renal.

Las evidencias actuales sugieren que ni la metformina ni los iSGLT2 deben iniciarse en pacientes con una TFGe $\leq 30 \mathrm{ml} / \mathrm{min} / 1.73 \mathrm{~m}^{2}$. Para los pacientes que inician un iSGLT2 con una TFGe $\geq 30 \mathrm{ml} / \mathrm{min} / 1.73$ $\mathrm{m}^{2}$ y que posteriormente disminuye a $<30 \mathrm{ml} / \mathrm{min} / 1.73$ $\mathrm{m}^{2}$, el iSGLT2 puede continuarse hasta el inicio de la terapia de reemplazo renal, de acuerdo con la estrategia valorada en el ensayo CREDENCE con canagliflozina ${ }^{35}$.

Recomendaciones

- En pacientes con DM2 y ERC se debe incluir un iSGLT2 como terapia de adición o monoterapia.

- En pacientes con DM2 y ERC se recomienda la adición de un iSGLT2 (preferentemente canagliflozina o dapagliflozina). En pacientes con DM con ECV y ERC el tratamiento debería elegirse en función de la evidencia para reducir eventos renales (caída de la TFGe del 50\%, progresión albuminuria, necesidad de terapia de sustitución renal) y disminución de eventos CV mayores y hospitalizaciones por IC, buscando un resultado más allá del efecto glucémico.
- Una vez que se ha iniciado el tratamiento con un iSGLT2, se considera razonable continuar cuando la TFGe disminuye a $<30 \mathrm{ml} / \mathrm{min} / 1.73 \mathrm{~m}^{2}$.

- En pacientes con TFGe $<30 \mathrm{ml} / \mathrm{min} / 1.73 \mathrm{~m}^{2}$, en general no debería iniciarse tratamiento con un iSGLT2, aunque de forma individualizada podría considerarse en pacientes con TFGe hasta $25 \mathrm{ml} / \mathrm{min} / 1.73 \mathrm{~m}^{2}$.

\section{Receptores de trasplante renal}

No hay evidencia suficiente que evalúe la eficacia y seguridad de los iSGLT2 en pacientes con trasplante de riñón, que pueden ser más vulnerables a infecciones debido a su estado inmunosuprimido. Solo se dispone de algunas series de casos o ensayos con iSGLT2 en pacientes postrasplante, que analizan su eficacia, resultados renales y seguridad. Los datos disponibles han demostrado que la adición de un iSGLT2 resulta en una disminución de la HbA1c, no hay deterioro de la función renal y el riesgo de infección urinaria recurrente es bajo $0^{40-43}$.

\section{Recomendaciones}

- En receptores de trasplante renal, Ios iSGLT2 son una opción de tratamiento, ya que han demostrado ser eficaces en la reducción de la HbA1c y no incrementan en riesgo de infecciones urinarias.

\section{Prevención de hipoglucemias}

La hipoglucemia es una de las consecuencias más temidas de los tratamientos antidiabéticos, y es uno de los principales factores limitantes para optimizar el control glucémico y para la adherencia terapéutica. La hipoglucemia se asocia con una considerable morbimortalidad, y es un posible factor de riesgo de deterioro neurocognitivo, de caídas y de lesiones relacionadas, además de causar un deterioro de la calidad de vida ${ }^{44}$.

La prevención de la hipoglucemia es un componente crítico del manejo de la DM. Los pacientes deben conocer las situaciones que aumentan su riesgo de hipoglucemia, así como aprender a reconocer los síntomas de esta ${ }^{11}$. En particular en los pacientes con enfermedad avanzada o ECV (incluida la IC), la prevención de la hipoglucemia es importante para reducir el riesgo de arritmias e isquemia miocárdica ${ }^{5}$.

Dado que el mecanismo de acción de los iSGLT2 es independiente de la vía de la insulina, son efectivos en todos los estadios de la DM2 y no producen 
hipoglucemia. Los iSGLT2 se aprobaron inicialmente para la DM2 como antidiabéticos sin mayor riesgo de hipoglucemia. Aunque el efecto hipoglucemiante de los iSGLT2 se atenúa con la TFGe reducida, puede ser necesario ajustar los agentes hipoglucemiantes concomitantes para evitar la hipoglucemia si se administran con insulina, sulfonilureas o glinidas. Debido a que los beneficios de la ERC y la ECV son en gran medida independientes de la dosis del iSGLT2, es preferible conservar la dosis de insulina con reducción de la dosis del iSGLT2 para mitigar el riesgo de cetoacidosis ${ }^{45}$.

Recomendaciones

- Los tres iSGLT2 disponibles en México han demostrado ser seguros con bajo riesgo de hipoglucemia en el paciente con DM.

\section{Efecto sobre el peso}

Los datos de los ensayos clínicos sugieren que los iSGLT2 producen una pérdida de peso promedio de aproximadamente 2 a $3 \mathrm{~kg}$ en pacientes con DM2, independientemente del tratamiento de base (monoterapia, como tratamiento complementario a otros agentes orales, e insulina) ${ }^{46}$.

El grado de pérdida de peso varía ligeramente según el agente y la dosis utilizada. Un metaanálisis de ensayos controlados aleatorizados que incluyeron participantes tratados con canagliflozina, dapagliflozina o empagliflozina, mostró reducciones significativas en el peso corporal vs. placebo para todos los tratamientos con iSGLT2: canagliflozina $300 \mathrm{mg}$, $-2.3 \mathrm{~kg}$ (IC 95\%: -2.6 a -1.9), empagliflozina $25 \mathrm{mg}$, $-2.2 \mathrm{~kg}$ (IC 95\%: -2.5 a -1.9) y dapagliflozina $10 \mathrm{mg}$, $-2.1 \mathrm{~kg}$ (IC 95\%: -2.5 a -1.8).

En comparación con otros fármacos hipoglucemiantes, los efectos de inhibición del SGLT2 variaron de una reducción de $-4.4 \mathrm{~kg}$ (IC 95\%: -4.8 a -4.1) para empagliflozina $25 \mathrm{mg}$ vs. sulfonilurea a -1.2 (IC $95 \%$ : -1.9 a -0.6$)$ para dapagliflozina $5 \mathrm{mg}$ vs. metformina (dapagliflozina $5 \mathrm{mg}$ no está disponible en México) ${ }^{47}$.

En datos de vida real, un estudio observacional en atención primaria demostró una pérdida media de peso de $2.6 \mathrm{~kg}$ 14-90 días después de comenzar con dapagliflozina y de $4.6 \mathrm{~kg}$ más allá de 180 días $^{48}$. La pérdida de peso con iSGLT2 generalmente se estabiliza después de 26 semanas de tratamiento en pacientes con DM2 a pesar de la excreción urinaria de glucosa sostenida ${ }^{46}$.

Recomendaciones

- Los iSGLT2 son la primera opción como terapia oral en adición a metformina en el escenario de
DM2 y obesidad, ya que brindan el beneficio de pérdida de peso (canagliflozina, dapagliflozina y empagliflozina).

- Aunque todos los iSGLT2 proporcionan pérdidas de peso de $2-3 \mathrm{~kg}$, el que mayor pérdida de peso ha demostrado es canagliflozina a la dosis de $300 \mathrm{mg}$ al día. Los iSGLT2 no están indicados como tratamiento de la obesidad o para inducir pérdida de peso.

\section{Seguridad}

Los iSGLT2 son fármacos con un buen perfil de tolerabilidad. Se han descrito algunos efectos secundarios.

\section{Infecciones genitourinarias}

El efecto secundario más común de los iSGLT2 es una mayor incidencia de infecciones micóticas genitales (principalmente balanitis y vulvovaginitis). Algunos estudios, aunque no todos, mostraron que estos fármacos también están asociados con un aumento discreto del riesgo de infecciones del tracto urinario. Estos efectos se atribuyen al aumento de glucosuria inducido por estos agentes. Generalmente, los eventos son de gravedad leve a moderada y no requieren la suspensión del fármaco. Las infecciones micóticas genitales son más prevalentes al inicio del tratamiento, tiende a remitir con el uso continuo y suelen resolverse con facilidad mediante el empleo de antifúngicos tópicos. Las infecciones del tracto urinario no complicadas pueden manejarse habitualmente con terapia estándar. Las infecciones del tracto urinario superior, como pielonefritis y urosepsis, son complicaciones extremadamente raras. Una infección micótica genital o depleción de volumen debe individualizarse de acuerdo con el escenario clínico, las preocupaciones del paciente y los posibles beneficios. Estos riesgos ocurren en toda la clase de iSGLT27,49.

Un metaanálisis de ensayos aleatorizados que compararon los iSGLT2 con placebo u otros medicamentos para la DM2 mostró que los iSGLT2 se asociaron significativamente con un aumento de cinco veces en el riesgo de infecciones micóticas genitales, sin incremento en el riesgo de infecciones urinarias ${ }^{50}$.

\section{Cetoacidosis diabética}

La cetoacidosis diabética (CAD) es un efecto adverso muy poco frecuente de los iSGLT2, no 
habiéndose demostrado hasta la fecha una relación causal entre la administración de un iSGLT2 y la aparición de cetoacidosis?.

Existen situaciones que podrían predisponer a la CAD, como deshidratación, restricción de la ingesta calórica, infecciones, cirugía, ingesta de alcohol y, sobre todo, reducción de la dosis de insulina, que se deben tener en cuenta a la hora de tratar al paciente con un iSGLT2?

La cetoacidosis euglucémica con elevación mínima o nula de los niveles de glucosa en sangre puede ocurrir en pacientes que toman iSGLT2 debido a un aumento de la oxidación de ácidos grasos y liberación de glucagón junto con una disminución de la secreción de insulina ${ }^{51,52}$.

El programa CVD-REAL (Comparative Effectiveness of Cardiovascular Outcomes in New Users of SodiumGlucose Cotransporter-2 Inhibitors) destacó este efecto secundario ${ }^{53}$, y de forma similar, un estudio en pacientes con DM que habían recibido una nueva prescripción de iSGLT-2 vs. iDPP-4 mostró cómo las tasas no ajustadas de CAD dentro de 180 días se duplicaban tras el inicio de iSGLT-2, con 4.9 eventos por 1,000 personas-año frente a 2.3 eventos por 1,000 personas-año, o una HR de 2.1 (IC 95\%: 1.5 a 2.9). Tras el ajuste en análisis pareado la HR se mantuvo en 2.2 (IC 95\%: 1.4 a 3.6) ${ }^{54}$.

Más recientemente, un estudio comparó los iSGLT2 frente a los iDPP-4 en nuevos consumidores entre los años 2013 y 2018 de varias bases de datos médicas de siete provincias del Canadá y Reino Unido y encontró una tasa de incidencia de CAD de 2.03 (IC 95\%: 1.83 a 2.25) en los iSGLT2 frente a 0.75 (IC 95\%: 0.63 a 0.89 ) de los iDPP-4, HR de 2.85 (IC 95\%: 1.99 a 4.08). La HR variaba según las moléculas: 1.86 (IC 95\%: 1.11 a 3.10) para dapagliflozina, 2.52 (IC 95\%: 1.23 a 5.14 ) para empagliflozina y 3.58 (IC 95\%: 2.13 a 6.03) para canagliflozina ${ }^{55}$.

Las fichas técnicas de los tres iSGLT2 comercializados en México advierten del riesgo aumentado de $C A D$, que puede presentarse de forma atípica y que debe considerarse en el caso de síntomas inespecíficos como náuseas, vómitos, anorexia, dolor abdominal, sed excesiva, dificultad respiratoria, confusión, fatiga o somnolencia inusuales.

\section{Amputaciones}

Es un efecto adverso muy poco prevalente ( $\geq 1 / 1,000 a<1 / 100$ eventos por 1,000 pacientes-año. La incidencia de amputaciones de miembros inferiores es muy baja en los ensayos y estudios observacionales en relación con el número de pacientes expuestos a iSGLT2. No obstante, parece prudente realizar exámenes regulares de los pies y evitar los iSGLT2 en aquellos pacientes con amputación previa o ulceración activa del pie ${ }^{56}$.

El estudio CANVAS ${ }^{26}$ mostró una incidencia de amputaciones de 0.6 eventos por 1,000 pacientes-año en el grupo de canagliflozina vs. 0.3 en el grupo placebo, aunque posteriormente el estudio CREDENCE ${ }^{28}$ no constató un incremento del riesgo de amputaciones entre los tratados con canagliflozina respecto al grupo tratado con placebo, con tasas de 12.3 vs. 11.2 por 1,000 pacientes-año en el grupo de canagliflozina y el grupo de placebo, respectivamente (HR: 1.11; IC 95\%: 0.79 a 1.56). La FDA retiró la advertencia en caja negra respecto al riesgo de amputaciones con canagliflozina por la nueva evidencia en estudios clínicos controlados y de vida real, donde este riesgo no estaba aumentado respecto a placebo.

El Observe 4D23, un estudio de vida real entre más de 700,000 pacientes con DM 2 tratados con diferentes antidiabéticos, comparó los efectos de los tratamientos en la hospitalización por IC y la incidencia de amputaciones de miembros inferiores por debajo de la rodilla. La comparación se realizó con canagliflozina $(150,000$ pacientes) frente a los otros iSGLT2 y otros antidiabéticos, ajustando por medio de propensity score, y no se observó aumento del riesgo de amputaciones con canagliflozina frente al resto de terapias. Los resultados fueron similares en pacientes con y sin ECV establecida ${ }^{57}$.

En la misma línea, un metaanálisis reciente de cinco estudios con iSGLT2 que evaluaron el riesgo de amputación ha concluido que el uso de iSGLT2 no se asoció con un aumento significativo en el riesgo de amputación en comparación con los controles ${ }^{58}$.

\section{Fracturas óseas}

Un metaanálisis de ensayos que evaluaron los resultados de seguridad combinados de canagliflozina, dapagliflozina y empagliflozina no apoyó un efecto dañino de los iSGLT2 sobre el hueso. La tasa de fracturas fue del $1.59 \%$ en el grupo de iSGLT2 y del $1.56 \%$ en el grupo de control. Además, la incidencia de eventos de fractura fue similar entre los tres iSGLT2 ${ }^{59}$.

\section{Depleción de volumen}

Los iSGLT2 reducen la presión arterial al inducir diuresis osmótica. Este efecto es beneficioso en personas 
con hipertensión no controlada, pero puede provocar mareos posturales, hipotensión ortostática y deshidratación, especialmente en personas de edad avanzada con enfermedad renal o que toman diuréticos de asa ${ }^{60}$.

En el estudio CANVAS ${ }^{26}$, la depleción de volumen fue más común en el grupo de canagliflozina frente a placebo (26 vs. 18.5 eventos por 1.000 personas-año; $p=0.009)$. Por el contrario, en el estudio DECLARETIMI $58^{29}$ el número de pacientes con acontecimientos indicativos de depleción del volumen fue equilibrado entre los grupos de tratamiento: $213(2.5 \%)$ y $207(2.4 \%)$ en los grupos de dapagliflozina y placebo, respectivamente. $Y$ en el ensayo EMPA-REG OUTCOME ${ }^{25}$ los eventos adversos compatibles con la depleción de volumen fueron raros y ocurrieron con una frecuencia similar en los grupos de empagliflozina y placebo (5.1 vs. $4.9 \%$, respectivamente).

Los iSGLT2 pueden causar una depleción de volumen debido a su efecto diurético. Sin embargo, por lo general no es necesario suspender o reducir las dosis de otros diuréticos al iniciar el iSGLT2, pero la monitorización de los niveles de electrolitos y la función renal es importante para la titulación de la dosis o el ajuste de otros agentes antihipertensivos o diuréticos. Para minimizar el riesgo de depleción de volumen, el tratamiento con iSGLT2 debe interrumpirse durante los periodos de enfermedad aguda u otros factores estresantes.

\section{Hiperpotasemia}

Algunos estudios han informado de un ligero aumento de los niveles séricos de potasio con los iSGLT2.

Las diferentes incidencias de hiperpotasemia en los ensayos con iSGLT2 posiblemente reflejen una diferencia en la combinación de casos de las cohortes del estudio (comorbilidades asociadas como insuficiencia renal) y el uso de fármacos que potencialmente afectan la homeostasis del potasio, como los inhibidores del sistema renina-angiotensina-aldosterona (SRAA). Por tanto, aunque el aumento de la concentración sérica de potasio es pequeño, parece indicado actuar con precaución y monitorizar el potasio en pacientes con insuficiencia renal, en pacientes que reciben medicamentos anti-SRAA concomitantes o en aquellos afectados por enfermedades que predisponen a la hiperpotasemia ${ }^{61}$.

Recomendaciones

- Se recomienda en pacientes con DM2 en tratamiento con sulfonilureas, con antecedente de hipoglucemia o alto riesgo de hipoglucemia, cambiar por un iSGLT2

- Se recomienda en adultos mayores con síndrome de fragilidad y uso de diuréticos de asa, el empleo de iSGLT2 con precaución e iniciando a dosis bajas.

- En pacientes con infecciones genitales, con isGLT2, se recomienda no interrumpir el tratamiento e iniciar tratamiento antifúngico.

- En pacientes con DM2 con antecedente de complicaciones agudas como cetoacidosis o estado hiperosmolar hiperglucémico con descontrol crónico e insulina, no se recomienda el empleo de iSGLT2 hasta mejorar el control metabólico.

\section{Lesión renal aguda}

En un metaanálisis de 18 ensayos clínicos se observó que los pacientes que consumen iSGLT2 se asociaron a una disminución del $24 \%$ en el riesgo de desarrollar lesión renal aguda, al compararse con iDPP4 y AR-GLP162, sustentado bajo la hipótesis de que disminuyen la hipoxia tubular generada por el alto consumo de oxígeno por el SGLT al absorber grandes cantidades de sodio y glucosa.

Recomendaciones

- Los ISGLT2 disminuyen el riesgo de lesión renal aguda y pueden considerarse como una alternativa terapéutica en pacientes con riesgo de padecer o que hayan padecido lesión renal aguda.

\section{Litiasis renal}

En una cohorte prospectiva de 2.3 años, se evidenció que aquellos pacientes que consumen iSGLT2 tuvieron una asociación a disminuir un $49 \%$ el riesgo de litiasis renal, en comparación a los AR-GLP1. Bajo la hipótesis de que los iSGLT2, al incrementar el volumen urinario por la diuresis osmótica que provocan, diluyen las moléculas litogénicas ${ }^{63}$.

\section{Recomendaciones}

- Los ISGLT2 disminuyen el riesgo de litiasis renal y pueden considerarse seguros en pacientes con DM2 y con riesgo de padecer o que hayan padecido litiasis renal.

\section{Uso de los iSGLT2 en pacientes de edad avanzada}

Se estima que el $30 \%$ de las personas mayores de 65 años cumplen los criterios diagnósticos de DM, 
siendo el 90\% DM2. Generalmente, las guías clínicas para el tratamiento de la DM2 en personas mayores abordan de manera muy limitada el uso de las terapias más recientes, como los iSGLT2, que tienen importantes beneficios para las personas mayores, como un bajo riesgo de hipoglucemia, reducción del riesgo CV y renal, y un mecanismo independiente de la insulina, lo que permite su uso durante toda la evolución de la $\mathrm{DM}^{60}$.

Si bien el deterioro de la función renal podría ser un factor limitante para su uso en la población anciana, la edad por sí misma no debería ser nunca una contraindicación, puesto que se ha demostrado su eficacia y seguridad incluso en mayores de 75 años. Sin embargo, es obligado aumentar las precauciones si se usa en pacientes de edad avanzada, por el mayor riesgo de deshidratación, trastornos hidroelectrolíticos, hipotensión e insuficiencia renal, secundarias a la diuresis osmótica, en este grupo de pacientes ${ }^{64}$.

La clase de los iSGLT2 se tolera bien, aunque también se sugiere cierta precaución, incluido el ajuste de terapias concomitantes, como insulina y antihipertensivos, especialmente diuréticos de asa ${ }^{60}$.

Los resultados observados dentro de los subgrupos de edad en los ensayos EMPA-REG OUTCOME ${ }^{25}$ y DECLARE-TIMI $58^{29}$ con empagliflozina y dapagliflozina, respectivamente, respaldaron la eficacia y seguridad de los iSGLT2 en poblaciones mayores con DM2. Es importante destacar que casi la mitad de los pacientes incluidos en DECLARE-TIMI 58 y EMPA-REG OUTCOME tenían más de 65 años.

Asimismo, los análisis combinados de ensayos clínicos aleatorizados mostraron una buena tolerabilidad de dapagliflozina y canagliflozina en pacientes de edad $\geq 65$ años $^{65}$.

La sólida evidencia de los beneficios CV y renales de los iSGLT2 ha llevado a las guías de tratamiento de la DM a recomendar el uso prioritario de los iSGLT2 sobre otras terapias antidiabéticas disponibles. La frecuencia de eventos adversos con el uso de iSGLT2 indica que no existen contraindicaciones para su introducción en el tratamiento de pacientes mayores, y se recomienda encarecidamente en aquellos con enfermedad CV y renal ${ }^{60}$.

Recomendaciones

- El uso de iSGLT2 en pacientes diabéticos de edad avanzada es seguro, pero se recomienda realizar un monitoreo adecuado para hacer ajustes a sus otros tratamientos con el fin de evitar cuadros de deshidratación, hipotensión arterial, etc.
- Es aconsejable tener la precaución de iniciar con la dosis más baja y, en caso de ser necesario, incrementarla.

- Hay que valorar el estado funcional del paciente y evitar prescribirlos en pacientes con síndrome de fragilidad.

\section{Uso de los iSGLT2 en embarazo}

No existen datos clínicos adecuados sobre el uso de iSGLT2 en el embarazo, pero estos medicamentos no se recomiendan sobre la base de estudios preclínicos que sugieren posibles efectos adversos durante las últimas etapas del desarrollo fetal.

\section{Recomendaciones}

- No se recomienda el uso de iSGLT2 en embarazo debido a la falta de evidencia.

\section{Financiamiento}

La realización de este trabajo de consenso fue auspiciada por un donativo no restringido al grupo de trabajo por Janssen, México. Las sugerencias presentadas en el siguiente documento son opinión de los autores y no representan una opinión o recomendación oficial de alguna sociedad o compañía editorial.

\section{Conflicto de intereses}

Los autores declaran que no existe conflicto de intereses.

\section{Responsabilidades éticas}

Protección de personas y animales. Los autores declaran que para esta investigación no se han realizado experimentos en seres humanos ni en animales.

Confidencialidad de los datos. Los autores declaran que en este artículo no aparecen datos de pacientes.

Derecho a la privacidad y consentimiento informado. Los autores declaran que en este artículo no aparecen datos de pacientes.

\section{Bibliografía}

1. Zheng Y, Ley SH, Hu FB. Global aetiology and epidemiology of type 2 diabetes mellitus and its complications. Nat Rev Endocrinol. 2018;14(2):88-98.

2. International Diabetes Federation. IDF Diabetes Atlas. Eight edition 2017 [internet]. International Diabetes Federation; 2017 [último acceso: abril 2021]. Disponible en: http://fmdiabetes.org/wp-content/uploads/2018/03/ IDF-2017.pdf 
3. Instituto Nacional de Estadística y Geografía e Instituto Nacional de Salud Pública. Encuesta Nacional de Nutrición y Salud 2018. Presentación de resultados [Internet]. México: Instituto Nacional de Estadística y Geografía e Instituto Nacional de Salud Pública. Disponible en: https:// ensanut.insp.mx/encuestas/ensanut2018/doctos/informes/ensanut_2018_presentacion_resultados.pdf

4. Basto-Abreu A, Barrientos-Gutiérrez T, Rojas-Martínez R, Aguilar-Salinas CA, López-Olmedo N, de la Cruz-Góngora V, et al. Prevalencia de diabetes y descontrol glucémico en México: resultados de la Ensanut 2016. Salud Publica Mex. 2020;62(1):50-9.

5. Cosentino F, Grant PJ, Aboyans V, Bailey CJ, Ceriello A, Delgado V, et al.; ESC Scientific Document Group. 2019 ESC Guidelines on diabetes, pre-diabetes, and cardiovascular diseases developed in collaboration with the EASD. Eur Heart J. 2020;41(2):255-323.

6. Hsia DS, Grove O, Cefalu WT. An update on sodium-glucose co-transporter-2 inhibitors for the treatment of diabetes mellitus. Curr Opin Endocrinol Diabetes Obes. 2017;24(1):73-9.

7. Miranda Fernández-Santos C, Egocheaga Cabello MI. Guía práctica de uso de los iSGLT2 en diabetes mellitus tipo 2 [Internet]. Sociedad Española de Médicos Generales y de Familia; 2020. Disponible en: https:// www.semg.es/images/documentos/guia_uso_iSGLT2.pdf

8. Ray KK, Seshasai SR, Wijesuriya S, Sivakumaran R, Nethercott $S$ Preiss D, et al. Effect of intensive control of glucose on cardiovascular outcomes and death in patients with diabetes mellitus: a meta-analysis of randomized controlled trials. Lancet. 2009;373:1765-72.

9. Turnbull FM, Abraira C, Anderson RJ, Byington RP, Chalmers JP, Duckworth WC, et al. Intensive glucose control and macrovascular outcomes in type 2 diabetes. Diabetologia. 2009;52:2288-98.

10. Laiteerapong N, Ham SA, Gao Y, Moffet HH, Liu JY, Huang ES, et al. The legacy effect in type 2 diabetes: impact of early glycemic control on future complications (the Diabetes \& Aging Study). Diabetes Care. 2019;42:416-26.

11. American Diabetes Association. Standards of Medical Care in Diabetes-2020. Diabetes Care. 2020;43(Suppl 1):S1-204.

12. Polidori D, Sha S, Mudaliar S, Ciaraldi TP, Ghosh A, Vaccaro N, et al. Canagliflozin lowers postprandial glucose and insulin by delaying intestinal glucose absorption in addition to increasing urinary glucose excretion: results of a randomized, placebo-controlled study. Diabetes Care. 2013;36(8):2154-61

13. Sha S, Polidori D, Farrell K, Ghosh A, Natarajan J, Vaccaro N, et al. Pharmacodynamic differences between canagliflozin and dapagliflozin: results of a randomized, double-blind, crossover study. Diabetes Obes Metab. 2015;17(2):188-97.

14. American Diabetes Association. Standards of Medical Care in Diabetes-2021. Diabetes Care. 2021;44(Suppl 1):S1-S232.

15. Lavalle-González FJ, Januszewicz A, Davidson J, Tong C, Qiu R, Canovatchel W, et al. Efficacy and safety of canagliflozin compared with placebo and sitagliptin in patients with type 2 diabetes on background metformin monotherapy: a randomised trial. Diabetologia. 2013; 56(12):2582-92

16. Schernthaner G, Gross JL, Rosenstock J, Guarisco M, Fu M, Yee J, et al. Canagliflozin compared with sitagliptin for patients with type 2 diabetes who do not have adequate glycemic control with metformin plus sulfonylurea: a 52-week randomized trial. Diabetes Care. 2013;36(9):2508-15. Erratum in: Diabetes Care. 2013;36(12):4172.

17. Bertoluci MC, Rocha VZ. Cardiovascular risk assessment in patients with diabetes. Diabetol Metab Syndr. 2017:9:25.

18. Wanner C, Lachin JM, Inzucchi SE, Fitchett D, Mattheus M, George J, et al. Empagliflozin and clinical outcomes in patients with type 2 diabetes mellitus, established cardiovascular disease, and chronic kidney disease. Circulation. 2018:137:119-29.

19. Verma S, McMurray JJV, Cherney DZI. The metabolodiuretic promise of sodium-dependent glucose cotransporter 2 inhibition: the search for the sweet spot in heart failure. JAMA Cardiol. 2017;2:939-40.

20. Sattar N, McLaren J, Kristensen SL, Preiss D, McMurray JJ. SGLT2 inhibition and cardiovascular events: why did EMPA-REG Outcomes surprise and what were the likely mechanisms? Diabetologia. 2016;59:1333-9.

21. Ferrannini E, Mark M, Mayoux E. CV protection in the EMPA-REG OUTCOME Trial: a "Thrifty Substrate" hypothesis. Diabetes Care. 2016;39:1108-14.

22. Baartscheer A, Schumacher CA, Wust RC, Fiolet JW, Stienen GJ, Coronel $\mathrm{R}$, et al. Empagliflozin decreases myocardial cytoplasmic $\mathrm{Na}+$ through inhibition of the cardiac $\mathrm{Na}+\mathrm{H}+$ exchanger in rats and rabbits. Diabetologia. 2017;60:568-73

23. Fedak PW, Verma S, Weisel RD, Li RK. Cardiac remodeling and failure from molecules to man (part II). Cardiovasc Pathol. 2005;14:49-60.

24. Packer M. Do sodium-glucose co-transporter-2 inhibitors prevent heart failure with a preserved ejection fraction by counterbalancing the effects of leptin? A novel hypothesis. Diabetes Obes Metab. 2018;20(6):1361-6.

25. Zinman B, Wanner C, Lachin JM, Fitchett D, Bluhmki E, Hantel S, et al. Empagliflozin, cardiovascular outcomes, and mortality in type 2 diabetes. N Engl J Med. 2015;373:2117-28.
26. Neal B, Perkovic V, Mahaffey KW, de Zeeuw D, Fulcher G, Erondu N, et al. Canagliflozin and cardiovascular and renal events in type 2 diabetes. N Engl J Med. 2017;377:644-57.

27. Mahaffey KW, Neal B, Perkovic V, de Zeeuw D, Fulcher G, Erondu N, et al.; CANVAS Program Collaborative Group. Canagliflozin for Primary and Secondary Prevention of Cardiovascular Events: Results from the CANVAS Program (Canagliflozin Cardiovascular Assessment Study). Circulation. 2018;137(4):323-34.

28. Perkovic V, Jardine MJ, Neal B, Bompoint S, Heerspink HJL, Charytan DM, et al. Canagliflozin and renal outcomes in type 2 diabetes and nephropathy. N Engl J Med. 2019;380:2295-306.

29. Wiviott SD, Raz I, Bonaca MP, Mosenzon O, Kato ET, Cahn A, et al. Dapagliflozin and cardiovascular outcomes in type 2 diabetes. N Engl J Med. 2019;380:347-57

30. Zelniker TA, Wiviott SD, Raz I, Im K, Goodrich EL, Bonaca MP, et al. SGLT2 inhibitors for primary and secondary prevention of cardiovascular and renal outcomes in type 2 diabetes: a systematic review and meta-analysis of cardiovascular outcome trials. Lancet. 2018;393(10166):319.

31. McMurray JJV, Solomon SD, Inzucchi SE, Kosiborod MN, Martinez FA, Ponikowski $\mathrm{P}$, et al. Dapagliflozin in patients with heart failure and reduced ejection fraction. N Engl J Med. 2019;381:1995-2008.

32. Packer M, Anker SD, Butler J, Filippatos G, Pocock SJ, Carson P, et al. Cardiovascular and renal outcomes with empagliflozin in heart failure. N Engl J Med. 2020; 383:1413-24.

33. Zannad F, Ferreira JP, Pocock SJ, Anker SD, Butler J, Filippatos G, et al. SGLT2 inhibitors in patients with heart failure with reduced ejection fraction: a meta-analysis of the EMPEROR-Reduced and DAPA-HF trials. Lancet. 2020;396(10254):819-29.

34. Cosentino F, Cannon CP, Cherney DZI, Masiukiewicz U, Pratley R, Dagogo-Jack S, et al.; VERTIS CV Investigators. Efficacy of ertugliflozin on heart failure-related events in patients with type 2 diabetes mellitus and established atherosclerotic cardiovascular disease: Results of the VERTIS CV Trial. Circulation. 2020;142(23):2205-15.

35. KDIGO 2020 Clinical Practice Guideline for Diabetes Management in Chronic Kidney Disease. Kidney Int. 2020;98(4S):S1-S115.

36. Wanner C, Inzucchi SE, Lachin JM, Fitchett D, von Eynatten M, Mattheus M, et al. Empagliflozin and progression of kidney disease in type 2 diabetes. N Engl J Med. 2016;375:323-34.

37. Neuen BL, Young T, Heerspink HJL, Neal B, Perkovic V, Billot L, et al. SGLT2 inhibitors for the prevention of kidney failure in patients with type 2 diabetes: a systematic review and meta-analysis. Lancet Diabetes Endocrinol. 2019;7:845-54

38. Heerspink HJL, Stefánsson BV, Correa-Rotter R, Chertow GM, Greene T, Hou FF, et al. Dapagliflozin in patients with chronic kidney disease. N Engl J Med. 2020;383:1436-46.

39. Cannon CP, Pratley R, Dagogo-Jack S, Mancuso J, Huyck S, Masiukiewicz $U$, et al. Cardiovascular outcomes with ertugliflozin in type 2 diabetes. N Engl J Med. 2020;383:1425-35.

40. AlKindi F, Al-Omary HL, Hussain Q, Al Hakim M, Chaaban A, Boobes Y. Outcomes of SGLT2 inhibitors use in diabetic renal transplant patients. Transplant Proc. 2020:52(1):175-8.

41. Mahling M, Schork A, Nadalin S, Fritsche A, Heyne N, Guthoff M. Sodium-glucose cotransporter 2 (SGLT2) inhibition in kidney transplant recipients with diabetes mellitus. Kidney Blood Press Res. 2019;44(5):98492.

42. Halden TAS, Kvitne KE, Midtvedt K, Rajakumar L, Robertsen I, Brox J, et al Efficacy and safety of empagliflozin in renal transplant recipients with posttransplant diabetes mellitus. Diabetes Care 2019; 42:106774.

43. Rajasekeran H, Kim SJ, Cardella CJ, Schiff J, Cattral M, Cherney DZI, et al. Use of canagliflozin in kidney transplant recipients for the treatment of type 2 diabetes: A case series. Diabetes Care. 2017;40:e75-e76.

44. Reyes-García R, Moreno-Pérez Ó, Tejera-Pérez C, Fernández-García D, Bellido-Castañeda V, de la Torre Casares ML, et al.; en representación del Grupo de trabajo de Diabetes-SEEN. Documento de abordaje integral de la diabetes tipo 2. Endocrinol Diabetes Nutr. 2019;66(7):443-58.

45. Tuttle KR, Brosius FC $3^{\text {rd }}$, Cavender MA, Fioretto P, Fowler KJ, Heerspink HJL, et al. SGLT2 inhibition for CKD and cardiovascular disease in type 2 diabetes: Report of a scientific workshop sponsored by the National Kidney Foundation. Diabetes. 2021;70(1):116.

46. Brown E, Wilding JPH, Barber TM, Alam U, Cuthbertson DJ. Weight loss variability with SGLT2 inhibitors and GLP1 receptor agonists in type 2 diabetes mellitus and obesity: Mechanistic possibilities. Obesity Reviews. 2019;20:816-28.

47. Zaccardi F, Webb DR, Htike ZZ, Youssef D, Khunti K, Davies MJ. Efficacy and safety of sodium-glucose co-transporter-2 inhibitors in type 2 diabetes mellitus: systematic review and network meta-analysis. Diabetes Obes Metab. 2016;18(8):783-94. 
48. Wilding J, Bailey C, Rigney U, Blak B, Beekman W, Emmas C. Glycated hemoglobin, body weight and blood pressure in type 2 diabetes patients initiating dapagliflozin treatment in primary care: a retrospective study. Diabetes Ther. 2016;7(4):6950711.

49. Lupsa BC, Inzucchi SE. Use of SGLT2 inhibitors in type 2 diabetes: weighing the risks and benefits. Diabetologia. 2018;61:2118-25

50. Rådholm K, Wu JH, Wong MG, Foote C, Fulcher G, Mahaffey KW, et al Effects of sodium-glucose cotransporter-2 inhibitors on cardiovascular disease, death and safety outcomes in type 2 diabetes - A systematic review. Diabetes Res Clin Pract. 2018;140:118-28.

51. Ogawa W, Sakaguchi K. Euglycemic diabetic ketoacidosis induced by SGLT2 inhibitors: possible mechanism and contributing factors. J Diabetes Investig. 2016;7:135-8

52. Rosenstock J, Ferrannini E. Euglycemic diabetic ketoacidosis: a predictable, detectable, and preventable safety concern with SGLT2 inhibitors. Diabetes Care. 2015;38:1638-42.

53. Kosiborod M, Lam CSP, Kohsaka S, Kim DJ, Karasik A, Shaw J, et al. CVD-REAL Investigators and Study Group. Cardiovascular events associated with SGLT-2 inhibitors vs. other glucose-lowering drugs: The CVD-REAL 2 Study. J Am Coll Cardiol. 2018;71(23):2628-39.

54. Fralick M, Schneeweiss S, Patorno E. Risk of diabetic ketoacidosis after initiation of an SGLT2 inhibitor. N Engl J Med. 2017:376:2300-2.

55. Douros A, Lix LM, Fralick M, Dell'Aniello S, Shah BR, Ronksley PE, et al Sodium-glucose cotransporter-2 inhibitors and the risk for diabetic ketoacidosis: A multicenter cohort study. Ann Intern Med. 2020;173(6):417-25.

56. Vardeny $\mathrm{O}$, Vaduganathan $\mathrm{M}$. Practical guide to prescribing sodium-glucose cotransporter 2 inhibitors for cardiologists. JACC Heart Fail. 2019;7(2):169-72

57. Ryan PB, Buse JB, Schuemie MJ, DeFalco F, Yuan Z Stang PE, et al. Comparative effectiveness of canagliflozin, SGLT2 inhibitors and nonSGLT2 inhibitors on the risk of hospitalization for heart failure and amputation in patients with type 2 diabetes mellitus: A real-world meta-analysis of 4 observational databases (OBSERVE-4D). Diabetes Obes Metab. 2018;20(11):2585-97.
58. Miyashita S, Kuno T, Takagi H, Sugiyama T, Ando T, Valentin N, et al. Risk of amputation associated with sodium-glucose co-transporter 2 inhibitors: A meta-analysis of five randomized controlled trials. Diabetes Res Clin Pract. 2020;163:108136.

59. Tang HL, Li DD, Zhang JJ, Hsu YH, Wang TS, Zhai SD, et al. Lack of evidence for a harmful effect of sodium-glucose co-transporter 2 (SGLT2) inhibitors on fracture risk among type 2 diabetes patients: a network and cumulative meta-analysis of randomized controlled trials. Diabetes Obes Metab. 2016;18:1199-206.

60. Custódio JS Jr, Roriz-Filho J, Cavalcanti CAJ, Martins A, Salles JEN. Use of SGLT2 Inhibitors in Older Adults: Scientific Evidence and Practical Aspects. Drugs Aging. 2020;37(6):399-409.

61. Cianciolo G, De Pascalis A, Capelli I, Gasperoni L, Di Lullo L, Bellasi A et al. Mineral and electrolyte disorders With SGLT2i Therapy. JBMR Plus. 2019;3(11):e10242.

62. Zhao M, Sun S, Huang Z, Wang T, Tang H. Network meta-analysis of novel glucose-lowering drugs on risk of acute kidney injury. Clin J Am Soc Nephrol. 2020;16(1):70-8.

63. Kristensen KB, Henriksen DP, Hallas J, Pottegård A, Lund LC. Sodium-glucose cotransporter 2 inhibitors and risk of nephrolithiasis. Diabetologia. 2021;64(7):1563-71.

64. Formiga F, Rodríguez-Mañas L, Gómez-Huelgas R. Papel de los inhibidores SGLT2 en el paciente anciano con diabetes mellitus; debemos evitar la discriminación por razón de edad. Rev Esp Geriatr Gerontol. 2016;51(6):307-8

65. Fioretto $P$, Mansfeld TA, Ptaszynska A, Yavin Y, Johnsson E, Parikh S. Long-term safety of dapaglifozin in older patients with type 2 diabetes mellitus: a pooled analysis of phase $\mathrm{Ilb} / \mathrm{II}$ studies. Drugs Aging. 2016;33(7):511-22.

66. Sinclair AJ, Bode B, Harris S, Vijapurkar U, Shaw W, Desai M, et al. Efcacy and safety of canagliflozin in individuals aged 75 and older with type 2 diabetes mellitus: a pooled analysis. J Am Geriatr Soc. 2016; 64(3):543-52. 